\title{
ESCAPE ROOM EDUCATIVA: CONCEPCIÓN DE LOS FUTUROS MAESTROS DE EDUCACIÓN SECUNDARIA EN ESPECIALIDAD DE EDUCACIÓN FÍSICA Y TECNOLOGÍA SOBRE LA EXPERIENCIA DE DISEÑAR Y PARTICIPAR EN UNA ESCAPE ROOM EDUCATIVA
}

\author{
Recepción: 26/08/2018 | Revisión: 22/02/2019 | Aceptación: 26/03/2019
}

\author{
Irene LÓPEZ SECANELL \\ Florida Universitaria \\ irelopsec@gmail.com
}

\author{
Enric ORTEGA TORRES \\ Florida Universitaria \\ eortega@florida-uni.es
}

\begin{abstract}
Resumen: En los últimos años se ha incrementado el interés por metodologías alternativas e innovadoras que poco a poco se van implementando en las aulas. Entre estas metodologías, encontramos la gamificación, con un componente lúdico y motivacional que paulatinamente ha ganado terreno en el ámbito educativo. Conscientes de ello, en el presente artículo se analiza la concepción de los futuros profesores de Educación Secundaria en especialidad de Educación Física $y$ Tecnología sobre la experiencia de diseñar y participar en una escape room educativa. Para ello, se muestran las propuestas didácticas desarrolladas en ambas materias y los resultados obtenidos de un cuestionario realizado con Google Forms. Los resultados muestran cómo los estudiantes valoran positivamente la experiencia de participar y diseñar una escape room a nivel educativo y ven viable su futura aplicación en el aula. Se muestra un ejemplo de investigación-acción que permite concluir la necesidad de transformar la formación inicial docente a través de estrategias como la gamificación y abrir líneas de investigación que apuesten por escape rooms inter y transdisciplinares.
\end{abstract}

Palabras clave: escape room; educación superior; formación inicial de maestros; propuesta didáctica, educación científica.

EDUCATIONAL ESCAPE ROOM: CONCEPTION OFTHE FUTURE PHYSICAL EDUCATION ANDTECHNOLOGY TEACHERS OF SECONDARY EDUCATION ONTHE EXPERIENCE OF DESIGNING AND PARTICIPATING IN AN EDUCATIONAL ESCAPE ROOM

\begin{abstract}
In recent years there has been an increase in the interest in alternative and innovative methodologies that are gradually being implemented in the classrooms. Among these methodologies, we find gamification, with a ludic and motivational component that has gradually gained ground in the educational field. Aware of this, this article analyzes the conception of future teachers of Secondary Education specializing in Physical Education and Technology on the experience of designing and participating in an educational escape room. Due to this, the didactic proposals developed in both subjects and the results obtained from a questionnaire made with Google Forms are shown. The results show how the students positively value the experience of participating and design an escape room in education and see their future application in the classroom as viable. This is an example of action research that allows to conclude the need to transform the initial teacher training through strategies such as gamification and opens lines of research that bet on inter and transdisciplinary escape rooms.
\end{abstract}

Keywords: escape room; higher education; initial teacher training; didactic proposal, science education.

\section{ESCAPE ROOM EDUCATIVA: CONCEPCIÓ DELS FUTURS MESTRES D'EDUCACIÓ SECUNDÀRIA D'ESPECIALITAT D'EDUCACIÓ FÍSICA ITECNOLOGIA SOBRE L'EXPERIĖNCIA DE DISSENYAR I PARTICIPAR EN UNA ESCAPE ROOM EDUCATIVA}

Resum: En els últims anys ha incrementat l'interès per metodologies alternatives i innovadores que a poc a poc es van implementant a l'aula. D'entre aquestes metodologies, trobem la gamificació, amb un component lúdic i motivacional que paulatinament ha guanyat terreny en làmbit educatiu. Conscients d'això, en aquest article s'analitza la concepció dels futurs mestres d'Educació Secundària d'especialitat en Educació Física i Tecnologia sobre l’experiència de dissenyar i participar en una escape room educativa. Amb aquest objectiu, mostrem les propostes didàctiques desenvolupades en ambdues matèries $\mathrm{i}$ els resultats obtinguts d'un qüestionari realitzat amb Google Forms. Els resultats mostren com els estudiants valoren positivament l'experiència de participar i dissenyar una escape room a nivell educatiu i veuen viable la seva futura implementació a l'aula. Es mostra un exemple d'investigació-acció que permet concloure la necessitat de transformar la formació inicial docent a través d'estratègies com la gamificació i obrir línies d'investigació que apostin per escape rooms inter i transdisciplinars.

Paraules clau: escape room; educació superior; formació inicial de mestres; proposta didàctica, educació científica. 


\section{Introducción}

La necesidad de actualización de los sistemas formativos es una urgencia que conlleva entender la educación como una parte importante del bien común (UNESCO, 2015). Son diversas las investigaciones educativas que evidencian como esta renovación debe dirigirse hacia la implantación de metodologías que fomenten un aprendizaje activo, donde el docente actúe como mediador y el estudiante participe con un rol activo en su proceso de aprendizaje (Fernández, 2006). Una metodologia activa implica que los estudiantes se involucren en su proceso de aprendizaje, que participen en clase y colaboren entre ellos. Además, se deben propiciar las condiciones que fomenten el pensamiento independiente y crítico a través de los análisis, las síntesis, los debates y la resolución de problemas basados en casos reales (Stanford. Teaching Commons, 2018; Stronge, 2002).

Junto a la necesidad de fomentar metodologías activas y participativas, se vislumbra la importancia de buscar formas de aprendizaje que fomenten la interdisciplinariedad entre diferentes saberes, lo cual nos aporta una perspectiva más amplia e interconectada para comprender la realidad compleja en la que vivimos. Morin (2006) defiende la necesidad de apostar por la interdisciplinariedad como una opción que permite unir, descubrir, asociar y problematizar los puntos de conexión entre los conocimientos. Por todo ello, es necesario crear una nueva concepción de escuela como espacio intercultural, donde el trabajo cooperativo e interdisciplinar entre el profesorado sea fundamental (Arandía, 2004).

En los últimos años se ha incrementado el interés por metodologías alternativas, innovadoras e interdisciplinares que poco a poco se van implementando en las aulas. Dentro de estas metodologías encontramos la gamificación, con un componente lúdico y motivacional que en los últimos años ha ganado terreno en el ámbito educativo. Conscientes de ello, en el presente artículo mostramos una experiencia práctica basada en una escape room como una estrategia de gamificación que favorece una metodología activa, participativa e interdisciplinar y que contiene algunos de los ingredientes necesarios para la transformación en la formación inicial docente. Para ello, el presente trabajo tiene el objetivo de analizar la concepción de los futuros profesores de educación secundaria en especialidad de Educación Física y Tecnología sobre la experiencia de participar y diseñar respectivamente una escape room educativa. Para ello empezaremos realizando una conceptualización teórica de los términos "gamificación" y "escape room", para dar las bases teóricas que nos permitan comprender el análisis realizado. Posteriormente se explicarán los métodos e instrumentos utilizados, junto al desarrollo de la propuesta didáctica. Esta propuesta didáctica se diferenciará en el proceso de diseño de una escape room por parte de los alumnos de la especialidad de tecnología del Máster de Secundaria y el proceso de participación en una escape room por parte de los alumnos de la especialidad de educación física. Finalmente se mostrarán los resultados y las conclusiones, donde se expondrán las limitaciones y futuras líneas de investigación.

\section{Escape room: características en relación con la gamificación}

En estas últimas décadas el desarrollo del videojuego ha crecido de forma exponencial y su investigación ha posibilitado detectar elementos del juego que pueden usarse para motivar compor- 
tamientos en otros campos. La gran mayoría de esos elementos ya se conocían anteriormente de juegos de azar, de cartas o de tablero, pero al convertirse en una industria tan enorme ha propiciado su estudio y análisis masivo, lo cual ha dado nueva información válida para entender sus componentes y usarlos entre otros medios en el aprendizaje.

La palabra "gamificación" es un anglicismo (gamification) que proviene de la palabra game (juego). En español se podría traducir como ludificación, si bien se está imponiendo el uso de su anglicismo. Este vocablo fue usado por primera vez en el año 2002 por Nick Pelling pero fue en 2010 cuando empezó a popularizarse al incorporar técnicas de juego en el ámbito educativo, debido a los numerosos beneficios que éste aporta a nivel cognitivo (Rodríguez, 2018).

Según Kapp (2012:9), la gamificación consiste en "la utilización de mecanismos, la estética y el uso del pensamiento para atraer, incitar a la acción, promover el aprendizaje y resolver problemas". Según Deterding, Dixon, Khaled y Nacke (2011) la gamificación se aplica en contextos donde comúnmente no hay juego. Por ello, en los últimos años la gamificación se ha aplicado en materias como Lengua, Ciencias o Matemáticas en distintos niveles educativos. En estas materias existen diversas estrategias para llevar a cabo la gamificación como pueden ser los cuentos motores, la teatralización, etc. En este artículo vamos a centrarnos en una estrategia que en los últimos años se ha ido extendiendo en el mundo educativo: la escape room.

El origen de la escape room está documentado en Japón en 2007 con la escape room creada por la agencia de publicidad SCRAP (Nicholson, 2015). En ésta un grupo de personas se encerraban en una habitación donde debían solucionar enigmas y distintas pruebas para ir resolviendo una historia y lograr escapar antes de que finalizara el tiempo disponible (generalmente de una hora). Cada escape room se ambientaba en temas, personajes e historias diferentes, lo cual hacia que la diversidad y el entretenimiento de estos espacios fuera muy amplia.

El planteamiento divertido, dinámico y entretenido de las escape rooms hizo que estas, poco a poco, se fueran adaptando en el ámbito educativo como una estrategia que facilitaba la creación de condiciones de aprendizaje basadas en el juego para favorecer la adquisición de ciertos contenidos curriculares. Desde un enfoque educativo, podemos definir la escape room como "una actividad que se desarrolla en un espacio cerrado donde un grupo de personas trabaja en equipo para resolver tareas y/o acertijos para poder salir de él." (Fernandez-Rio, 2018:2). Nicholson (2015) complementa la definición enfatizando que las escape room son juegos basados en el trabajo en equipo donde los jugadores deben descubrir pistas, resolver enigmas, puzles y/o tareas con el fin de alcanzar un objetivo final en un tiempo limitado para poder salir de una estancia cerrada. Aunque en los últimos años las publicaciones sobre experiencias de escape room se han incrementado, aún son pocas las experiencias publicadas que puedan ser transferibles a otros contextos. Entre las últimas experiencias más destacadas encontramos la publicación de Monaghan y Nicholson (2017), quienes implementan una escape room en estudios de patofisiología o Hita y Sánchez (2017) quienes utilizan la realidad virtual en una escape room con estudiantes del grado de ingeniería informática. 


\section{Método e instrumentos}

\subsection{Participantes}

La práctica presentada se ha llevado en el Máster Universitario en Profesorado de Educación Secundaria con el alumnado de las especialidades de Educación Física (1 grupo de 16 alumnos) y la especialidad de Tecnología ( 2 grupos con un total de 41 alumnos).

\subsection{Instrumentos}

Los instrumentos que se han empleado para recoger información sobre la percepción del alumnado acerca de la ejecución de la propuesta didáctica analizada en este artículo se basan en un cuestionario post en formato Google Forms formado por 6 preguntas de respuesta múltiple cerrada (RM) y 4 de respuesta abierta (RA) para que el alumnado participante realice una valoración crítica de la ejecución de la practica escape room educativa y sobre su utilidad didáctica y otras 2 preguntas (RI) más con el fin de identificar la especialidad del estudiante y su identidad,

En el cuestionario se realizan varias preguntas con respuesta múltiple cerrada en una escala Likert de 4 respuestas. SÍ, si, no, NO, siendo Sí la de mayor grado de acuerdo y NO la de menor. A continuación, se muestra el cuestionario utilizado para la recogida de la información.

1. RA ¿Qué contenidos has aprendido en la escape room?

2. RA ¿Para qué te ha servido realizar la escape room?

3. RA ¿Cuáles son los aspectos que más valoras de haber realizado la escape room?

4. RA ¿Qué mejorarías de la escape room?

5. $\mathrm{RM}_{i}$ Consideras que una escape room es una buena estrategia para generar aprendizaje con el alumnado de secundaria?

6. $\mathrm{RM}_{¿}$ Cuál es la característica de una escape room que provoca aprendizaje en el alumnado?

7. $\mathrm{RM}_{\dot{Q}}$ Qué tipo de competencia es la que más se pone en juego en una escape room?

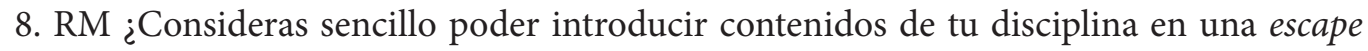
room educativa?

9. RM Una escape room educativa es una buena oportunidad para planificar juntamente con otros docentes o mejor para una única disciplina.

10. RM En la elaboración de una unidad didáctica, ¿consideras la opción de introducir un escape room educativa?

11. RI ¿Cuál es la especialidad de tu Máster?

12. RI Nombre 


\subsection{Análisis de la información}

El proceso llevado a cabo para la recogida y análisis de la información fue establecido de mutuo acuerdo entre los docentes de ambas especialidades. En primer lugar, se realizó el diseño de las secuencias didácticas de la intervención a realizar, y se acordó pasar un cuestionario realizado conjuntamente por ambos docentes en la última sesión del curso para ambas especialidades. El pase de los cuestionarios se estructuró con una explicación previa de los objetivos del cuestionario y se dejó un tiempo de respuesta de 20 minutos, permitiendo realizar respuestas a posteriori dejando abierto el enlace durante 15 días posteriores a la finalización del curso. Se mandaron varios mensajes recordatorios para recoger el máximo número de respuestas a lo largo del periodo de 15 días. El alumnado participante debía identificarse para asegurar la validez de las respuestas y la no duplicidad

Los datos recogidos en los cuestionarios en formato Google Forms fueron exportados a Excel con el fin de realizar los posteriores análisis basados en la agrupación temática de las respuestas a preguntas abiertas y posterior diseño de las representaciones gráficas mostradas en el apartado de resultados.

\subsection{Desarrollo de la propuesta didáctica \\ 2.4.1 Escape room en Educación Física}

El objetivo de aprendizaje de la escape room era revisar los contenidos de educación física trabajados hasta la fecha mediante una escape room. Los contenidos que se pretendían revisar eran los siguientes: tipologías de evaluación, el constructivismo, la fórmula Karvonen y la expresión corporal. Para dar respuesta al objetivo y a los contenidos planteados se planteó una Escape room en un aula. La escape room se desarrollaba entorno a una historia basada en un personaje inventado llamado "Sedentario" que nos encerraba en llave en el aula para no poder salir y realizar actividad física. Mediante una performance realizada por la profesora disfrazada de "Sedentario" se les explicaba a los futuros maestros de Educación Física que tenían la misión de salir de la prisión del aula superando unas pruebas.

Para ello debían abrir una caja que "Sedentario" había cerrado con un candado de cuatro cifras que guardaba la llave del aula. El ganador tenía algunos privilegios para una actividad que posteriormente realizaríamos en el gimnasio, la cual se basaba en la elaboración de actividades basadas en los distintos tipos de estilos de enseñanza en educación física.

La organización de la actividad consistía en 4 grupos de 4 estudiantes y un total de 4 retos. La actividad fue planteada para realizarla en un total de 45 minutos.

Los retos fueron organizados de tal forma que ninguno de los grupos pudiera coincidir en una prueba. En el anexo 1 se especifican los enunciados de cada uno de los retos y, a continuación, se describe más detalladamente cada uno de éstos:

- Crucigrama: Una de las cuatro pruebas consistía en realizar un crucigrama que se basaba en contenidos de educación física que habíamos trabajado durante el curso sobre la evaluación y el constructivismo. El crucigrama estaba formado por distintas columnas e hileras vacías. En el 
crucigrama se marcaban dos casillas con dos colores distintos: naranja y azul. Una vez rellenado el crucigrama, los alumnos podían descubrir a qué letras correspondían cada uno de los colores indicados. Con las letras debían utilizar una leyenda donde cada letra se correspondía a un número. Debían anotar el número en la casilla de solución (Figura 1), escribiendo primero el número correspondiente al color naranja y luego al azul. Finalmente obtenían un número de cuatro dígitos que les abría una caja con las instrucciones para la siguiente prueba. Además, si sumaban sus cifras obtendrían el primer número que abre el candado de la última caja.
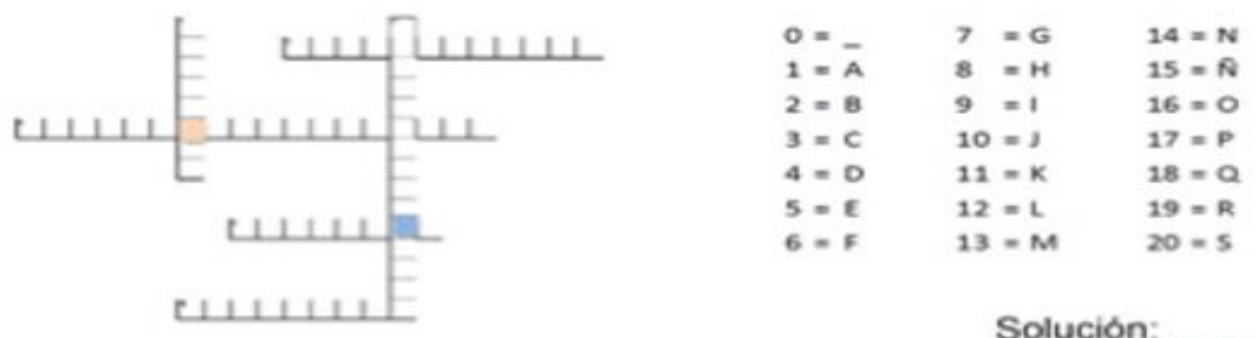

\section{Solución:}

Figura 1. Diseño del crucigrama utilizado en la escape room. Fuente: Propia.

-Twister: Los estudiantes disponían de unas instrucciones para jugar al Twister. El Twister estaba en el suelo y consistía en unos círculos de felpa con los colores típicos del juego original siguiendo este orden: amarillo, verde, azul y rojo. Había 4 columnas y 6 hileras. Las hileras se indicaban con un número al inicio de cada una. En cambio, cada una de las columnas se identificaba con un dibujo de una figura geométrica en la parte superior siguiendo este orden: cuadrado, $\mathrm{X}$, triángulo, círculo.

Los estudiantes tenían que seguir las órdenes que se les indicaba en un folio y colocar la mano o el pie correspondiente en el círculo del color que se indicaba (Figura 2). Con la ultima indicación tenían que observar la figura que se creaba con los cuerpos y descubrir que se dibujaba el segundo número que abría el candado de la última caja. Además, ese mismo número abría una nueva caja que tenían en la mesa con unas indicaciones para realizar una prueba nueva.

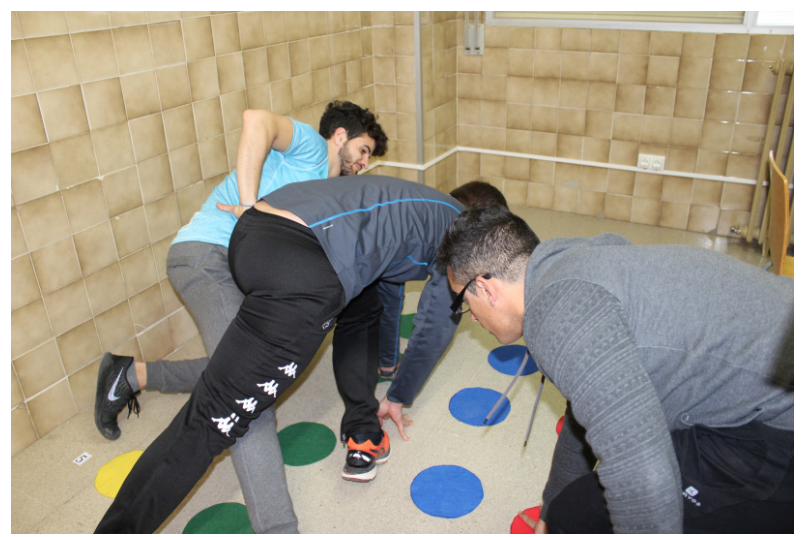

Figura 2. Alumnos realizando una da las pruebas de la escape room. Fuente: Propia. 
-QRs: La tercera prueba consistía en resolver un problema basado en la fórmula de karvonen a través de códigos QR. Los alumnos recibían un mensaje que decía que tenían que buscar un mensaje escondido en algún lugar de la clase. Este se escondía debajo de una silla. La silla no se podía mover, lo cual obligaba a los estudiantes a leer y resolver el problema debajo de la silla, estirados en el suelo. El problema consistía en utilizar la fórmula de Karvonen. La formula de Karvonen tiene en cuenta la frecuencia cardíaca máxima (FCmax) y la frecuencia cardíaca en reposo (FCrep) para calcular un porcentaje determinado de esfuerzo. La fórmula para calcularla es la siguiente:

$$
\% \text { de FCobjetivo }=((\text { FCmax }- \text { FCrep }) \times \% \text { intensidad })+\text { FCrep }
$$

En la prueba se les proponía calcular la frecuencia cardíaca de una persona de 50 años que quiere entrenar a un 70\%. Para completarlo les faltaba información que debían obtener con dos pruebas. La primera consistía en un folio con distintos QR ordenados intencionadamente. Cada alumno disponía de un lector de QR en su móvil, con el cual debían ir descubriendo cada código. Cada QR correspondía a una letra. Al final, las letras que acababan conformando la palabra "sesenta y cinco", la cual correspondía a la FCrep.

La segunda prueba consistía en descubrir un número secreto escondido en una tabla con distintas coordenadas (Figura 3).

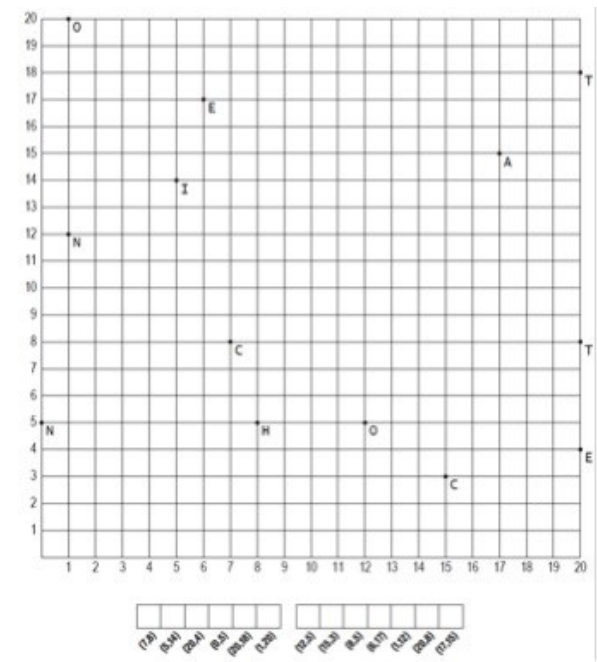

Figura 3. Diseño de una prueba de la escape room. Fuente: Propia.

Para descubrir el número tenían que leer las coordenadas que se les indicaban a una leyenda situada debajo de la tabla. De esta forma, si la leyenda era $(7,8)$, el 7 correspondía al eje X (horizontal) y el 8 al eje Y (vertical). Debían encontrar el punto de intersección entre el eje X y Y indicado. En ese punto se les mostraba una letra que debían escribir al recuadro que se encontraba encima de la leyenda.

La palabra secreta era "Ciento ochenta" que era la FCmax que necesitaban para la fórmula 
Karvonen. Con esta información ya tenían todos los datos para realizar la operación y obtener los latidos por minuto: 145. Este número abría una caja que tenían sobre la mesa, la cual les daba el tercer número del candado final y las instrucciones para la siguiente prueba.

- Globos: La última prueba consistía en una caja con muchos globos deshinchados. Cada uno de los globos tenía un mensaje escrito en un papel que se encontraba en el interior del globo. Los alumnos tenían que hinchar los globos hasta encontrar uno que tenía un número escrito en letras (Figura 4). Si el globo que hinchaban no era el correcto debían atarlo y estallarlo en parejas o grupo con alguna parte de su cuerpo.
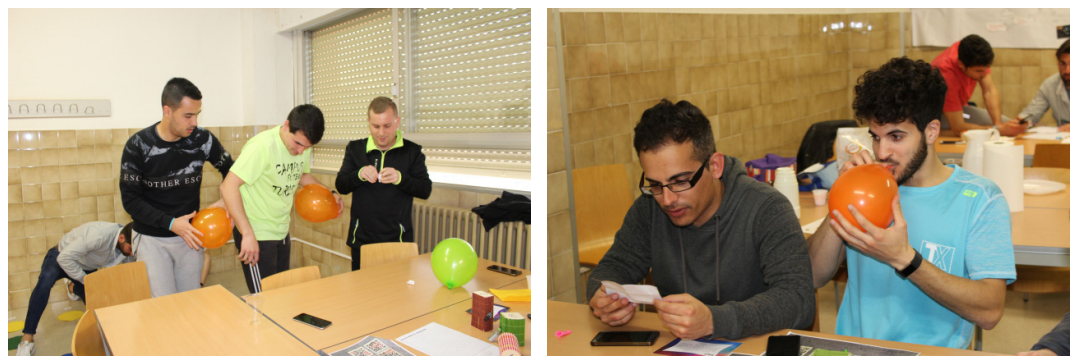

Figura 4. Alumnos resolviendo la prueba con globos. Fuente: Propia.

Al final, un grupo de alumnos consiguió la clave del candado y pudo abrir la caja donde se escondía la llave que abría el aula. Además, sin olvidar el objetivo de la escape room, el ganador tenía algunos privilegios para la actividad que posteriormente realizaríamos en el gimnasio, la cual consistía en la elaboración de actividades basadas en los distintos tipos de estilos de enseñanza.

\subsubsection{Escape Tech Room}

La intervención didáctica llevada a cabo en la modalidad de Tecnología del Máster de Secundaria se realizó tras haber formado al alumnado en el concepto de proyectos STEM, entendiendo el desarrollo de dichos contenidos a partir de los resultados obtenidos en el proyecto europeo Steam $4 \mathrm{U}^{1}$ y desde la definición de alfabetización STEM establecida por Couso (2017: 24): "Estar alfabetizado en STEM es ser capaz de identificar y aplicar, tanto los conocimientos clave como las formas de hacer, pensar, hablar y sentir de la ciencia, la ingeniería y la matemática, de forma más o menos integrada, para comprender, decidir y/o actuar ante problemas complejos y para construir soluciones creativas e innovadoras, aprovechando las sinergias personales y las tecnologías disponibles, de forma crítica, reflexiva y con valores".

El objetivo de la escape room en la modalidad de tecnología se centraba en conseguir que el alumnado diseñara una propuesta didáctica donde se desarrollaran contenidos de los proyectos STEM a través de una escape room que conectara las pruebas diseñadas por cada subgrupo formado por 4/6 alumnos. Para llevarla a cabo se dedican 2 sesiones de $4 \mathrm{~h}$ para diseñar, redactar la memoria de la propuesta didáctica y para montar los artefactos STEM para que pudieran ponerse

1 Consultar "www.steam4u.eu” 
a prueba en un grupo de $3^{\circ}$ de ESO. La propuesta realizada es el trabajo final de la asignatura Complementos para la formación disciplinar en la especialidad de Tecnología del Master de Secundaria y es evaluada y calificada por el docente a través de la rúbrica adjunta en el anexo 3. Los criterios y pesos que integra la rúbrica son: Justificación (25\%), Planificación (25\%), Evaluación (25\%), Innovación (10\%), Calidad (10\%), Prototipo (5\% ).

La falta de publicaciones sobre experiencias de gamificación en el ámbito educativo y la falta de formación del profesorado han obligado a crear unas orientaciones para su aplicación en el aula. Por ello, basándonos en Steinprinz, Kronberga y Salgado (2017), los estudiantes han tenido en cuenta los siguientes procedimientos para elaborar su escape room:

1. Seleccionar y plantear los objetivos de aprendizaje. Estos deben estar conectados con los intereses de los alumnos.

2. Seleccionar los contenidos que se quieren trabajar.

3. Diseño de los retos a resolver. Es importante que los retos tengan características variadas y que impliquen niveles de ejecución distintos.

4. Programar el orden de los distintos retos. Una buena organización favorecerá que los alumnos sean más efectivos a la hora de tomar decisiones (Fernandez-Rio, Sanz, Fernández-Cando y Santos, 2017).

5. Definir la organización del aula.

6. Crear la historia o narrativa en que se desarrollará la actividad.

En la imagen siguiente (Figura 5), podemos ver a los alumnos del máster durante el proceso de elaboración de los artefactos que completaban la propuesta didáctica realizada.

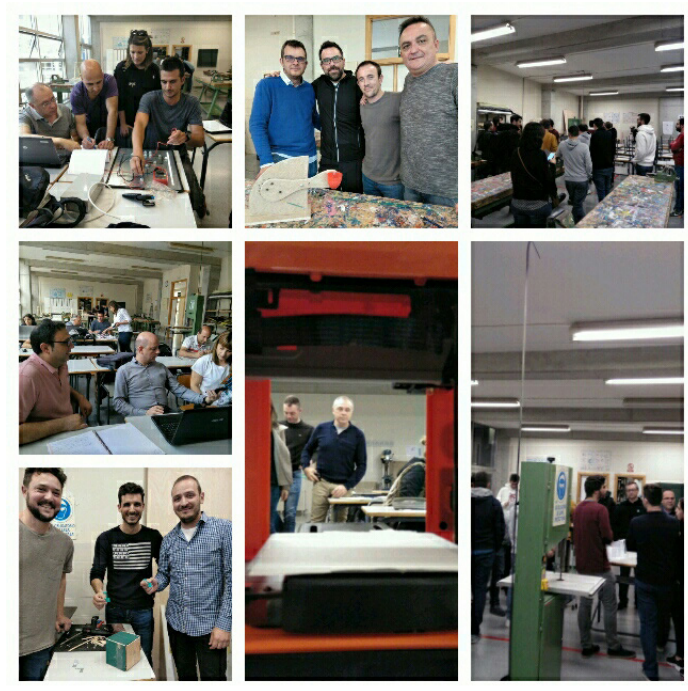

Figura 5. Alumnado participante. Fuente: Propia. 
La propuesta de actuación para el alumnado venia descrita conforme a las indicaciones que se exponen en el anexo 2 .

Los objetivos plateados en dicha actividad estaban centrados en:

1. Conseguir que el alumnado del Máster realizara el diseño de una propuesta didáctica en equipo mediante una dinámica de trabajo cooperativo para evitar que cada subgrupo elaborara su propia prueba sin establecer comunicación con el resto de los grupos. Este objetivo se establece a partir de la necesidad expuesta en las clases teóricas de la asignatura sobre la importancia de trabajar en equipo que se requiere en los claustros de centros de secundaria.

2. Realizar un diseño adaptado a un nivel de ESO concreto para acercar al alumnado del máster a la realidad y dificultad que conlleva planificar una secuencia didáctica para un grupo específico. Es por ello por lo que cada grupo debía de planificar una prueba de la Escape room que debía ser realizable para el alumnado de la ESO y suponer un mini-reto que pusiera a prueba algún conocimiento STEM integrado en la asignatura de Tecnología del currículo de $3^{\circ}$ de Secundaria

3. Asegurar que el alumnado del Máster conociera, a través de su integración en la propuesta didáctica, los contenidos de la asignatura de Tecnología en la ESO analizados en sesiones anteriores.

4. Conocer los materiales y equipamiento del taller de tecnología de un centro de secundaria. Con este objetivo se pide a cada grupo que se realice el diseño de un artefacto prototipo para complementar los materiales didácticos a entregar al alumnado de ESO para la realización de la prueba de escape room.

5. Finalmente, también se establece el objetivo aprender a realizar el diseño de materiales educativos dirigidos al alumnado de ESO y de este modo conseguir que se entienda desde la aplicación práctica la diferencia entre los documentos requeridos para una programación de aula y los entregados al alumnado para su ejecución (Ver anexo 2).

En la Figura 6 podemos ver alguno de los artefactos construidos por los diferentes grupos de alumnos del máster. 

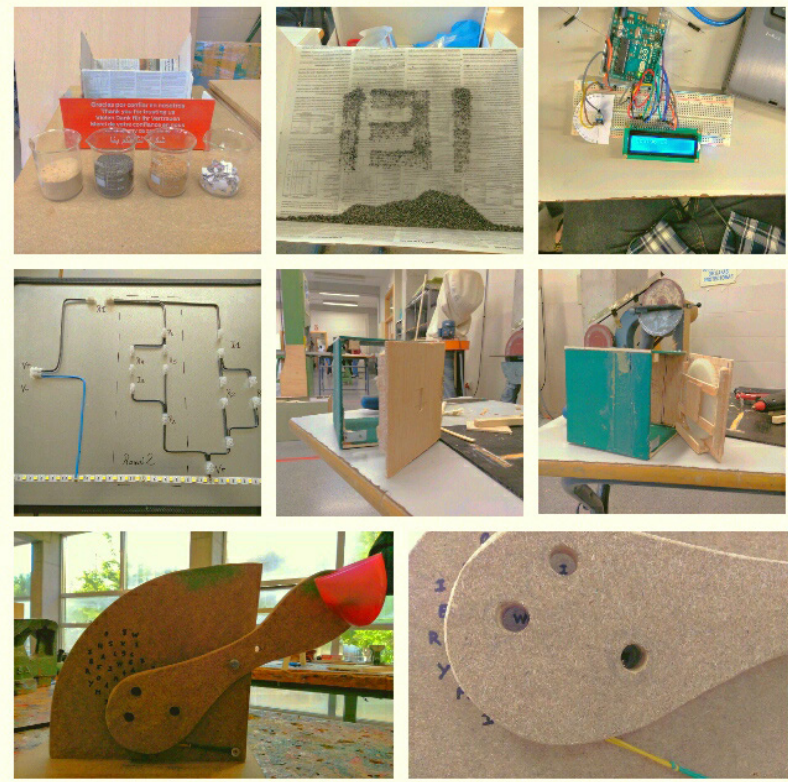

Figura 6. Imágenes de los artefactos construidos para la Escape Tech Room. Fuente: Propia.

A partir de las instrucciones dadas y las sesiones dedicadas a su ejecución, se entregaron 6 pruebas distintas para la Escape Tech Room global:

1. Prueba 1: Circuito eléctrico: Se entrega al alumnado un conjunto de resistencias varias para que pueda montar un circuito eléctrico con unas condiciones dadas. La resolución otorga al alumnado un código final que sirve para iniciar otra prueba.

2. Prueba 2: Triángulos con Arduino: Se plantean problemas sobre triángulos a resolver mediante Teorema de Pitágoras. La resolución va otorgando al alumnado una serie de códigos que sirven para finalizar el código de Arduino que sirve para poner en funcionamiento un circuito electrónico.

3. Prueba 3: Materiales: Se entrega al alumnado diferentes materiales para que encuentren un código escondido que se obtiene solamente con material ferromagnético por su combinación con imanes.

4. Prueba 4: Balanza: Se plantean una serie de problemas matemáticos la resolución de los cuales muestra al alumnado el número de elementos que deben ubicar en una balanza para que en su estado de equilibrio muestre un código escondido.

5. Prueba 5: Cerradura impresora 3D: Se entregan tres llaves diferentes al alumnado y mediante el cálculo de su volumen deben escoger la que abre una cerradura que esconde un mensaje.

6. Prueba 6: Arduino Leds: Mediante la resolución de problemas lógicos se van encontrando los códigos que deben incorporarse a la programación en código de un arduino que una vez finalizado ilumina de forma correcta un código de colores con LED. 
En la siguiente tabla se muestran los contenidos principales integrados en cada una de las pruebas que forman la Escape Tech Room:

\begin{tabular}{|c|c|c|c|c|}
\hline $\begin{array}{c}\text { PRUEBAS } \\
\text { ESCAPE ROOM }\end{array}$ & $\begin{array}{c}\text { CONTENIDOS } \\
\text { FÍSICA } \\
\mathrm{S}\end{array}$ & $\begin{array}{c}\text { CONTENIDOS } \\
\text { INGENIERIA } \\
\text { T }\end{array}$ & $\begin{array}{c}\text { CONTENIDOS } \\
\text { TECNOLOGIA } \\
\text { E }\end{array}$ & $\begin{array}{c}\text { CONTENIDOS } \\
\text { MATEMÁTICAS } \\
\text { M }\end{array}$ \\
\hline $\begin{array}{l}\text { PRUEBA 1: } \\
\text { CIRCUITO } \\
\text { ELÉCTRICO }\end{array}$ & LEY DE OHM & & $\begin{array}{c}\text { USO DEL } \\
\text { POLÍMETRO }\end{array}$ & \\
\hline $\begin{array}{l}\text { PRUEBA 2: } \\
\text { TRIÁNGULOS } \\
\text { CON ARDUINO }\end{array}$ & LEY DE OHM & & $\begin{array}{l}\text { MAGNITUDES } \\
\text { ELÉCTRICAS }\end{array}$ & $\begin{array}{l}\text { TRIGONOMETRIA } \\
\text { PROBLEMAS } \\
\text { GEOMETRÍA } \\
\text { (TEOREMA } \\
\text { PITÁGORAS) }\end{array}$ \\
\hline PRUEBA 3: & $\begin{array}{l}\text { PROPIEDADES } \\
\text { MATERIALES } \\
\text { PROBLEMAS } \\
\text { ENERGIA, } \\
\text { DENSIDAD, } \\
\text { FUERZAS }\end{array}$ & & & $\begin{array}{c}\text { PROBLEMAS } \\
\text { ÁLGEBRA } \\
\text { CÁLCULO } \\
\text { VOLUMEN FIGURAS } \\
\text { IRREGULARES }\end{array}$ \\
\hline $\begin{array}{l}\text { PRUEBA 4: } \\
\text { BALANZA }\end{array}$ & $\begin{array}{l}\text { USO DE LA } \\
\text { BALANZA }\end{array}$ & DISEÑO 3D & & $\begin{array}{l}\text { PROBLEMAS } \\
\text { ALGEBRA }\end{array}$ \\
\hline $\begin{array}{l}\text { PRUEBA 5: } \\
\text { CERRADURA } \\
\text { IMPRESORA 3D }\end{array}$ & $\begin{array}{l}\text { MEDIDAS DE } \\
\text { VOLUMEN }\end{array}$ & $\begin{array}{c}\text { USO IMPRESORA } \\
\text { 3D }\end{array}$ & & $\begin{array}{l}\text { GEOMETRIA } \\
\text { FIGURAS } \\
\text { REGULARES }\end{array}$ \\
\hline $\begin{array}{c}\text { PRUEBA 6: } \\
\text { ARDUINO LEDS }\end{array}$ & & USO ARDUINO & $\begin{array}{c}\text { LENGUAJE } \\
\text { PROGRAMACIÓN } \\
\text { CIRCUITO } \\
\text { ELECTRÓNICO }\end{array}$ & \\
\hline
\end{tabular}

Tabla 1. Contenidos desarrollados en la Escape Tech Room. 


\section{Resultados}

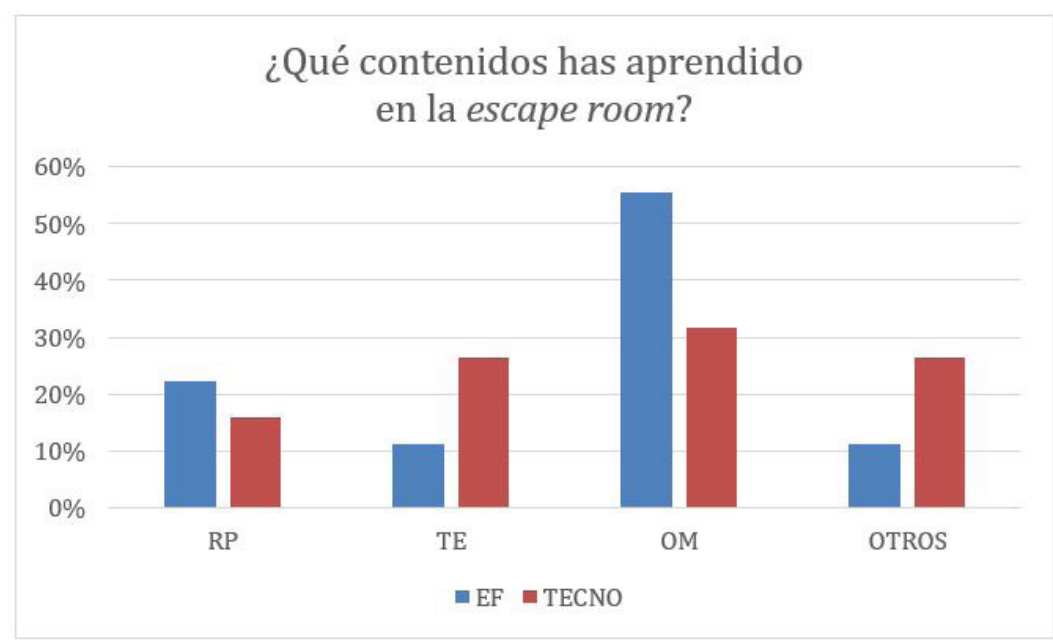

Figura 7. Resultados sobre los contenidos aprendidos. Fuente: Propia.

La pregunta “¿Qué contenidos has aprendido en la escape room?” Se formuló en modo abierto con el fin de obtener todas las versiones de respuesta que los alumnos pudieran responder. A pesar de tratarse de dos modalidades de intervención diferentes según la modalidad, la del alumnado de la especialidad de Educación Física centrada en la participación en la escape room y la de la especialidad de Tecnología centrada en el diseño, las respuestas de ambos grupos se pudieron agrupar fácilmente en tres grandes grupos por ser respuestas cortas y referirse a un solo contenido. La agrupación se realizó según el concepto que se exponía. Encontramos que la respuesta mayoritaria fue la que se refería a el aprendizaje de otras metodologías (OM) a través de la escape room en ambas modalidades, seguido de la consideración sobre haber aprendido a desarrollar el trabajo en equipo (TE) y la resolución de problemas (RP) con porcentajes diferentes según la modalidad. El resto de las respuestas (OTROS) no eran agrupables por su diversidad.

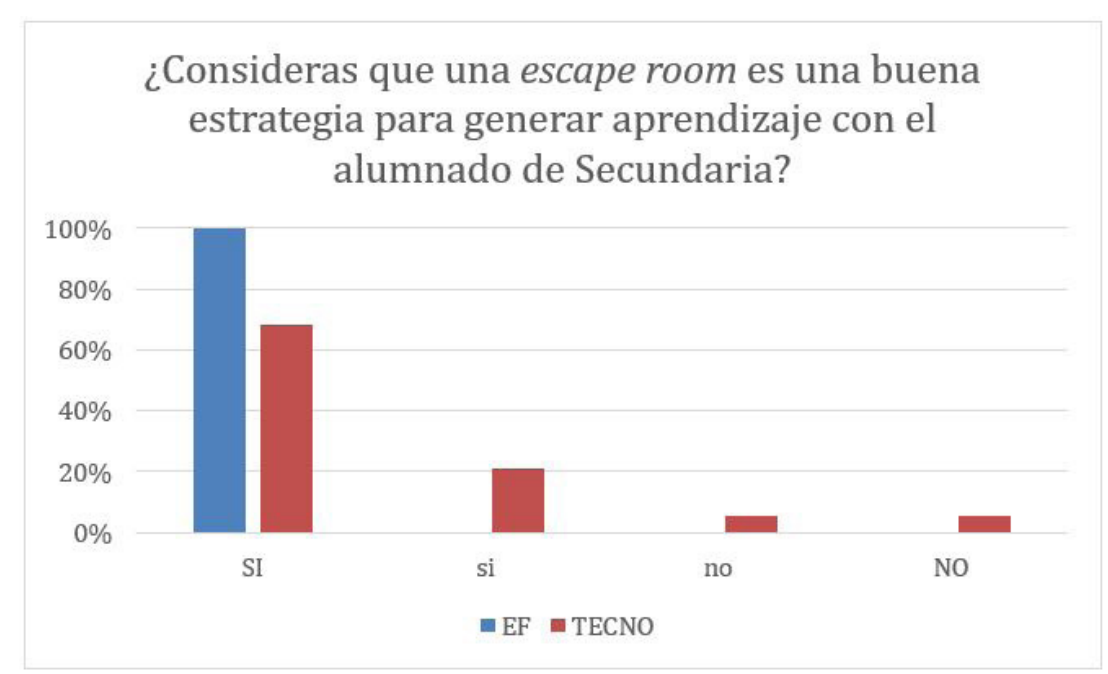

Figura 8. Resultados sobre la utilidad de la escape room. Fuente: Propia. 
Respecto a las respuestas obtenidas en las preguntas cerradas con opción múltiple destaca el grado de acuerdo sobre la consideración de buena estrategia el desarrollo de una escape room en el aula de secundaria para generar aprendizaje tal y como se aprecia en la Figura 8. Con un porcentaje del 100\% en la especialidad de Educación Física y del 89\% en la de Tecnología.

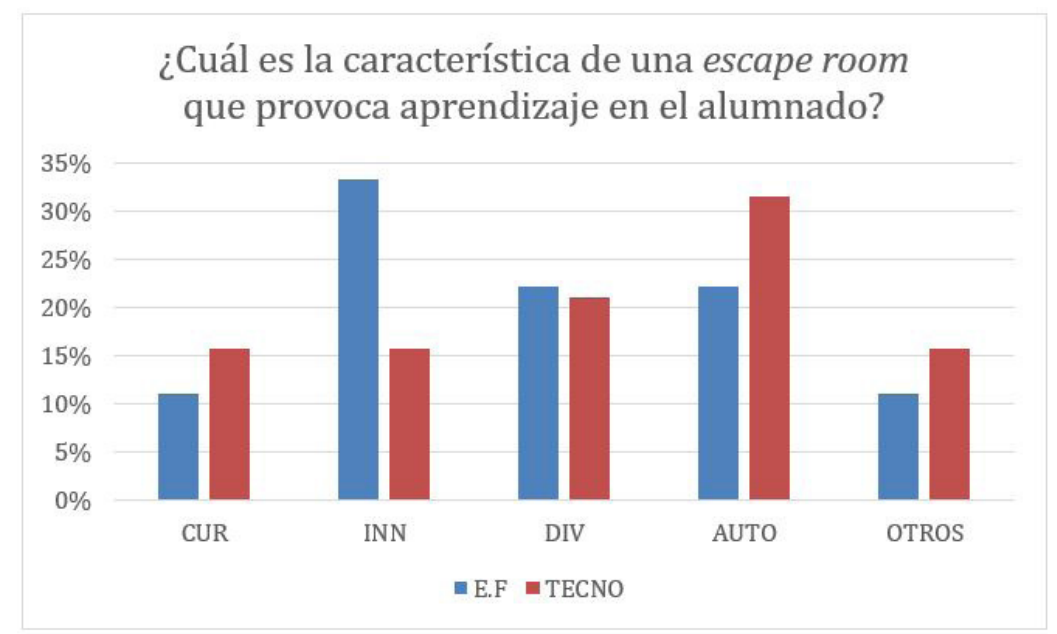

Figura 9. Resultados sobre las características de la Escape room. Fuente: Propia.

Con el fin de profundizar en la respuesta anterior, se pide al grupo de alumnos participante que destaque una característica de la escape room que sea generadora de aprendizaje. Las respuestas expuestas en la Figura 9 muestran un porcentaje muy similar para la categoría de "aprendizaje debido a la diversión (DIV)” en ambos casos, (21\%-22\%)) además destacan las respuestas que hacían referencia al aprendizaje debido a la innovación (INN) con una consideración mucho mayor para la especialidad de EF (33\% frente a 15\%) y a la autonomía en su ejecución (AUTO) con un porcentaje de consideración mayor para la especialidad de Tecnología (33\% frente a 22\%). También destaca la consideración de la curiosidad (CUR) como factor de aprendizaje importante para ambas modalidades con porcentajes mayores al 10\% en ambos casos. Existen otras respuestas no agrupables debido a su diversidad y baja frecuencia.

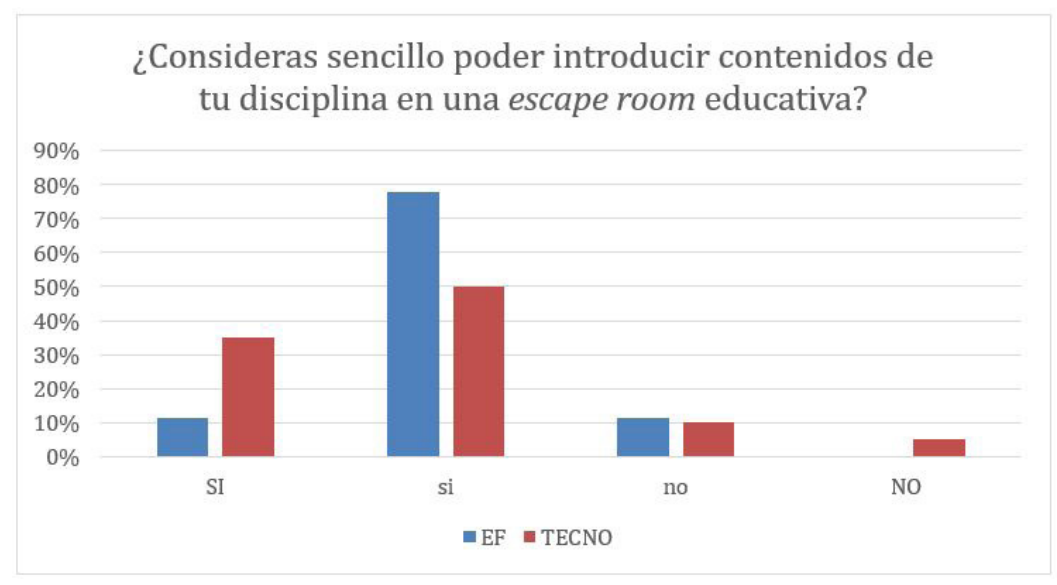

Figura 10. Resultados sobre la opinión de los estudiantes sobre la incorporación de la escape room en su disciplina. Fuente: Propia. 
En cuanto a la facilidad de integrar contenidos de la propia disciplina en una propuesta de escape room para secundaria se aprecia en la Figura 10 que existe un mayor grado de concepción de facilidad para el alumnado de Tecnología a pesar de que ambas modalidades consideran sencilla dicha posibilidad.

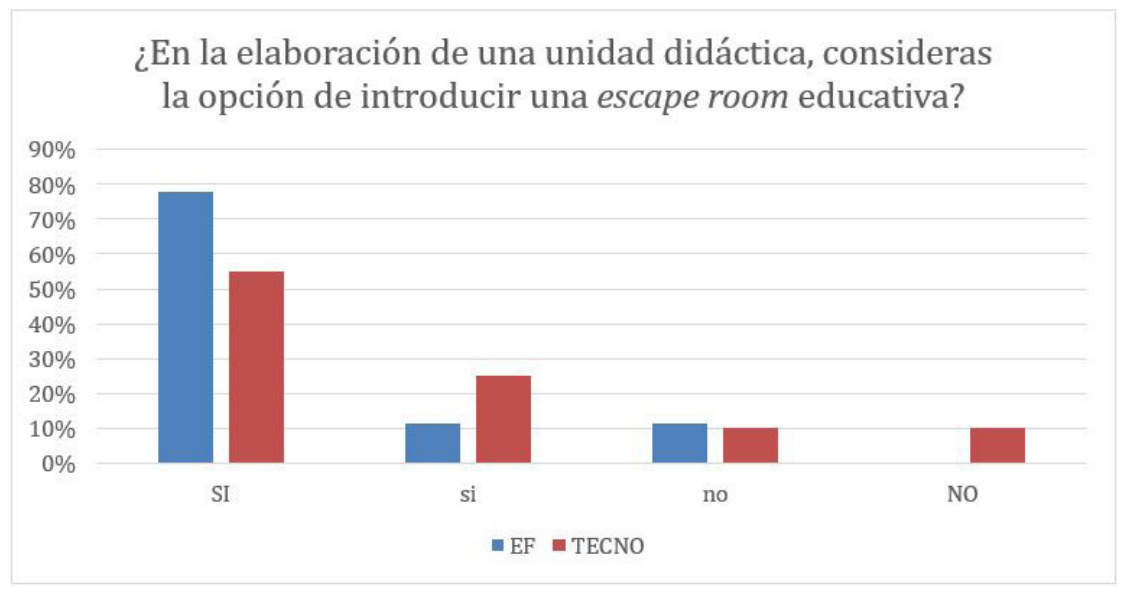

Figura 11. Resultados sobre la opinión de los estudiantes sobre la incorporación del escape room en la unidad didáctica. Fuente: Propia.

Cuando se pregunta al alumnado del Máster de Secundaria si consideraría la posibilidad de introducir en la unidad didáctica que deben desarrollar dentro de las asignaturas del Máster una propuesta de escape room educativa destaca el alto grado de intención de realizarlo por los alumnos de ambas modalidades. Como se aprecia en la figura 11 en ambos casos el porcentaje de alumnos que no consideran esta opción es muy bajo (11\% frente a $20 \%$ ).

\section{Conclusiones}

El presente artículo tenía la finalidad de analizar la concepción de los futuros profesores de educación secundaria en las especialidades de educación física y tecnología sobre la experiencia de participar y diseñar respectivamente una escape room educativa.

La práctica realizada y la información obtenida a partir de las opiniones del alumnado participante nos llevan a concluir que el desarrollo de una escape room educativa, tanto en el formato de protagonistas o como diseñadores de ésta, es una experiencia didáctica bien recibida y valorada por los futuros profesores. Junto a la valoración positiva de la experiencia, los resultados obtenidos también evidencian que los futuros maestros ven viable la aplicación de escape rooms en sus especialidades. Además, manifiestan cómo éstas han favorecido su aprendizaje en nuevas metodologías y trabajo en equipo, lo cual nos abre nuevas líneas de investigación sobre la incidencia de las escape rooms en estos factores.

Pese a tener una visión basada en su vivencia como estudiantes, los futuros docentes muestran una predisposición alta a experimentar con nuevas metodologías. Esta buena predisposición y valoración positiva por parte de los futuros docentes, pone en evidencia la responsa- 
bilidad que tenemos sus formadores de llevar a cabo prácticas alternativas bien ejecutadas, con realismo y contraste previo, que puedan servir para que los futuros docentes las integren en su futuro banco de recursos.

El presente estudio tiene una limitación en el diseño del cuestionario, ya que se evalúa con una misma batería de preguntas a poblaciones cuya experiencia con la escape room educativa es distinta. Para ello, para futuras investigaciones se propone involucrar al alumnado de tecnología y educación física tanto en el diseño como en la participación en una escape room realizada conjuntamente. De esta forma no solamente reforzaríamos la interdisciplinariedad, sino que estaríamos enfocando la práctica hacia la transdisciplinariedad. En la interdisciplinariedad, las materias actúan independientemente y desde cada una de ellas se realizan conexiones con contenidos que no son propios de la materia en cuestión. La transdisciplinariedad se ocupa de lo que hay "entre", "a través" y "más allá" de las disciplinas (Nicolescu, 1996). De esta forma, se crean relaciones entre los contenidos y profesores de distintas materias implicadas en un mismo proyecto común, lo cual nos permite comprender mejor la complejidad de la realidad desde un trabajo colaborativo.

Es desde la propia experiencia práctica como el futuro docente debe entender la importancia del aprendizaje activo y para ello sus formadores debemos llevar a cabo un cambio profundo en las metodologías utilizadas en docencia universitaria. El cambio al que tratamos de empujar a nuestro alumnado con el fin que se conviertan en docentes del siglo XXI debe empezar por una transformación propia de nuestra práctica docente, sin ello seguiremos teorizando sobre la necesidad de transformar las metodologías educativas, pero sin ser ejemplo de ello.

\section{Referencias bibliográficas}

Arandía, M. (2004). La formación de educadores y educadoras desde la mirada de Freire. Revista Interuniversitaria de Formación del Profesorado, 18(2), 59-77.

Couso, D. (2017). Per a què estem a STEM? Un intent de definir l'alfabetització STEM per a tothom i amb valors. Ciències: Revista del Professorat de Ciències de Primària i Secundària, 34, 22-30. Deterding, S., Dixon, D., Khaled, R., y Nacke, L. (2011). Gamification: towards a definition. Recuperado de http://gamification-research.org/wp-content/uploads/2011/04/02Deterding-Khaled-Nacke-Dixon.pdf

Fernandez-Rio, J. (2018). De los desafíos cooperativos a las edicoop-Escape rooms. XI Congreso Internacional de Actividades Física Cooperativas. Recuperado de https:/goo.gl/R1MGPo

Fernandez-Rio, J., Sanz, N., Fernández-Cando, J., y Santos, L. (2017). Impact of a sustained cooperative learning intervention on student motivation. Physical Education and Sport Pedagogy, 22(1), 89-105.

Fernández, M. R. (2006). Reseña de "Metodología participativa en la enseñanza universitaria" de Fernando López Noguero. Revista Interuniversitaria de Formación del Profesorado, 20(3), 313-316. 
Hita, A., y Sanchez, C. (2017). Desarrollo de una escape room mediante tecnologías de realidad virtual para aplicaciones de formación. (Tesis doctoral, Universitat Politècnica de Catalunya, España). Recuperado de https://goo.gl/koY9Cd

Kapp, K. M. (2012). The gamification of learning and instruction: game-based methods and strategies for training and education. San Francisco: John Wiley \& Sons.

Morin, E. (2006). Articular los saberes. ¿Qué saberes enseñar en las escuelas? México: Centre National de la Documentation Pédagogique (CNDP).

Monaghan, R., y Nicholson, S. (2017). Bringing escape room concepts to pathophysiology case studies. Journal of the Human Anatomy and Physiology Society, 21(2), 49-62.

Nicholson, S. (2015). Peeking behind the locked door: A survey of escape room facilities. Recuperado de https://goo.gl/1eaC7G

Nicolescu, B. (1996). La transdisciplinarité. manifeste. Monaco: Éditions du Rocher.

Rodríguez, C. A. C. (2018). Gamificación en educación superior: experiencia innovadora para motivar estudiantes y dinamizar contenidos en el aula. Edutec. Revista Electrónica de Tecnología Educativa, (63), 29-41.

Stanford. Teaching Commons (2018). Promoting active learning. Recuperado de https://goo.gl/ HNfoTa

Steinprinz, G., Kronberga, R., y Salgado, I. (2017). Eduesc@peroom. Manual para educadores. Recuperado de https://goo.gl/VTFzoD

Stronge, J. H. (2002). Qualities of effective teachers. Alexandria: Association for Supervision and Curriculum Development.

UNESCO (2015). Rethinking education towards a global common good? Recuperado de https:// goo.gl/AE6XXs 